\title{
Non-lethal method for the preparation of metaphase spreads using cultured mantle tissue from live adult abalone
}

\author{
Jun Hyung Ryu ${ }^{1}$, Sang Yoon Lee², Yoon Kwon Nam ${ }^{1,2}$ \& Seung Pyo Gong ${ }^{*, 1,2}$ \\ ${ }^{1}$ Department of Fisheries Biology, Pukyong National University, Busan 48513, Korea, ${ }^{2}$ Department of \\ Marine-Biomaterials \& Aquaculture, Pukyong National University, Busan 48513, Korea
}

BioTechniques 65: 33-36 (July 2018) 10.2144/btn-2018-0014

Keywords: adult abalone $\bullet$ culture $\bullet$ karyotyping $\bullet$ mantle $\bullet$ metaphase spreads

Metaphase spread preparation in adult abalone has not been successful, which has restricted the applications of karyotyping-based technologies. Here, we present a non-lethal method to enable preparation of metaphase spreads from live adult abalone using a tissue culture method. Mantle tissue fragments from live adult abalone were cultured in vitro and the cultured cells were used for metaphase spread preparation. To retrieve a sufficient number of proliferating cells required for metaphase spread preparation, at least 14 days of culture was required, and culturing the marginal zone of mantle was more optimal than culturing other areas. Additionally, it was shown that simple medium consisting of basal medium, fetal bovine serum and antibiotics could stimulate cellular proliferation followed by metaphase spread preparation.

Abalone (Haliotis discus hannai) is a commercially important marine gastropod that is a valuable food source [1] and, thus, several artificial breeding technologies such as hybrid fertilization, polyploid production and selective breeding have been developed to increase its productivity and quality [2]. To verify the technical fulfilment of these artificial breeding technologies, karyotyping should be able to be performed in live adult abalone to which these technologies have been applied. However, unlike in mammals and fish, karyotyping in live adult gastropods is not easy due to the difficulty in retrieving a sufficient number of proliferating cells. In gastropods, the cells that are directly retrieved from adult somatic tissues are not suitable for karyotyping as they contain very few proliferating cells $[3,4]$. Additionally, the specific mitogens that act on gastropods have not yet been verified [5] and an appropriate tissue culture method has not been established [6,7]. This indicates that a method to induce the proliferation of somatic cells from abalone should be developed to accomplish karyotyping in live adult abalone. Moreover, although a few studies have reported the successful karyotyping of adult abalone, all of them required sacrifice of the animals in order to isolate target tissues and did not provide any information regarding the efficiency of metaphase spread preparation $[3,4,8]$. This indicates that a well-organized method without animal sacrifice is required.

Mantle is the dorsal body wall covering visceral mass and its main function is to secrete the organic matrix and the calcium and carbonate ions required for biomineral precipitation for a shell [9]. Owing to its protrusive structure [10], removing small pieces of tissue from it does not influence the survival and growth of abalone and the impaired epithelium in incision sites can recover in 2 months even though the sites are not filled with granulation tissue [11]. Indeed, all abalone that provided small pieces of mantle in our study survived and did not show any abnormal sign in their growth during the 6-month observation period after tissue sampling. Hence, with the aim of using the mantle as a tissue donor for metaphase spread preparation without animal sacrifice, this article reports an efficient method to be able to prepare metaphase spreads in live adult abalone by retrieving a sufficient number of proliferating cells through tissue culture. For this, we retrieved mantle tissue fragments from live adult abalone, cultured them, prepared metaphase spreads from the cultured cells and determined the optimal culture period, tissue area for culture and medium composition on the basis of the efficiency of metaphase spread preparation.

The research was exempted from the need for review by the Institutional Animal Care and Use Committee (IACUC) of Pukyong National University (Busan, Korea) since the use of invertebrate is not the subject of review. Nevertheless, all experimental procedures dealing with the animals complied with the ethical guidelines published by the International Council for Laboratory Animal Science (ICLAS).

We established a new and efficient method to prepare metaphase spreads from adult abalone without animal sacrifice by providing an efficient way to induce cellular proliferation in mantle tissue culture. Our method introduces the optimal culture period, the optimal tissue area and a simplified medium composition for the culture of mantle tissue fragments. 


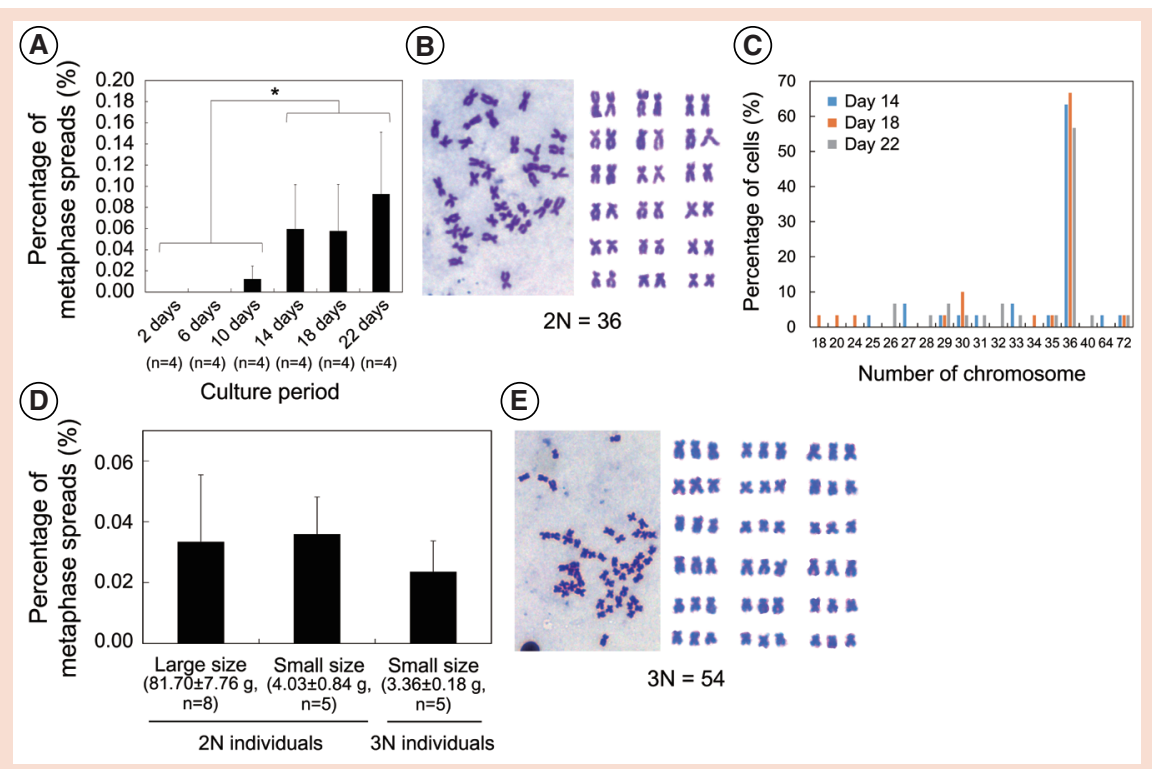

Figure 1. Examination of the optimal culture period for metaphase spread preparation by mantle tissue culture. Mantle tissue fragments were cultured in complete media in an $18^{\circ} \mathrm{C}$ incubator in air and the cultured cells were used for metaphase spread preparation. (A) Percentage of metaphase spreads according to culture period. Metaphase spreads were not observed until day 6 of culture beginning to appear from day 10 of culture. Asterisks indicate significant difference between two pooled groups cultured for 2-10 days and for 14-22 days ( $p=0.028$, t-test). (B) A representative image of normal diploid metaphase spread $(2 \mathrm{~N}=36)$ derived from the cell cultured for 14 days. (C) Percentage of cells containing a normal or abnormal number of chromosomes according to culture period. Percentages of the cells that have normal chromosome number were $63.3,66.7$ and $56.7 \%$ in the cells cultured for 14, 18 and 22 days, respectively. (D) Percentage of metaphase spreads within mantle cells cultured for 14 days when the mantle cells were obtained from abalone of different size and ploidy. No significant difference was observed among the groups. (E) A representative image of triploid metaphase spread $(3 \mathrm{~N}=54)$. For $(\mathbf{A})$ and $(\mathbf{D})$, data are mean \pm SEM from the replicates of " $n$ " times.

As shown in Figure 1A, significantly higher percentages of metaphase spreads were observed in the groups cultured for 14 , 18 and 22 days compared with those for 2 , 6 and 10 days $(p=0.028)$. Normal diploid metaphase spreads were easily identified (Figure 1B; $2 \mathrm{~N}=36$ ) and the proportion of them in 30 metaphase spreads was similar between the groups cultured for 14 , 18 and 22 days (Figure 1C; 56.7-66.7\%). From these experiments, we confirmed that metaphase spreads can be prepared efficiently when the culture persisted for 14 to 22 days. Hereafter, the culture period in all experiments was 14 days unless otherwise stated.

To test if this condition can be applied to other abalones that have different size and ploidy, three abalone groups including large diploid, small diploid and small triploid abalone, were subjected to metaphase spread preparation under the same condition as above. Triploid abalone were artificially produced and grown in the Genetics \& Breeding Research Center of the National Fisheries Research and Development Institute (Geoje, Korea). No significant difference was detected in the percentage of metaphase spreads between large and small diploid abalone $(0.033 \pm 0.022$ vs $0.036 \pm 0.012 \%)$ as well as between diploid and triploid small abalone $(0.036 \pm 0.012$ vs $0.024 \pm 0.010 \%)$ (Figure 1D). In case of triploid abalone, chromosome number was 54 as expected (Figure 1E; 3N =54). This demonstrates that this condition can be applied regardless of abalone size and ploidy state.

Next, we investigated the effects of mantle areas on metaphase spread preparation. Mantle tissues were divided into three areas: central zone (CZ), sub-marginal zone (SMZ) and marginal zone (MZ) (Figure $2 A)$. As shown in Figure 2B, when the culture aspects of each tissue fragments were observed, proliferation and outward migration of the cells from tissue fragments began from day 2 in $C Z$ and day 6 in SMZ and $\mathrm{MZ}$ after culture, and it continued to day 22 regardless of mantle areas. However, the scale of these phenomena was relatively high in $S M Z$ and $M Z$ compared with $\mathrm{CZ}$ and, in between $\mathrm{SMZ}$ and $M Z$, little difference was observed. Concerning the percentage of metaphase spreads, MZ showed a significantly higher value compared with $S M Z(p=0.013)$, but did not show a difference compared with CZ (Figure 2C). However, percentage of trials in which metaphase spread appeared was significantly higher in MZ (75\%, 9/12) compared with both CZ (25\%, 3/12) and SMZ (17\%, 2/12) (Figure 2D). The proportions of normal diploid metaphase spreads in 30 samples were 40, 50 and $50 \%$ in $\mathrm{CZ}, \mathrm{SMZ}$ and MZ, respectively (Figure 2E). These data indicate that $\mathrm{MZ}$ is the optimal area in mantle for metaphase spread preparation through mantle tissue culture.

Based on our previous report regarding abalone cell culture [12], we thus far used complex media designated as "Leibovitz's L-15 (L15) complete" containing fetal bovine serum $(F)$, hemolymph $(H)$, $\beta$-mercaptoethanol $(B)$, nonessential amino acids $(\mathrm{N})$, sodium selenite $(\mathrm{S})$, basic fibroblast growth factor, epidermal growth factor, leukemia inhibitory factor, abalone embryo extract, medaka embryo extract and antibiotics in L15 media. To simplify this, we finally examined the effects of medium supplements on metaphase spread preparation. MZ-derived mantle tissue fragments were cultured under six media designated as L15, L15F, L15H, L15FH, L15FHBNS and L15 complete. Details of each media are shown in Supplementary Table 1. When tissue fragments were cultured in L15F, L15FHBNS and L15 complete, significant increases in the percentage of metaphase spreads were observed compared with the L15 group (Figure 2F; $p=0.021$ ). Percentages of trials in which metaphase spread appeared showed a similar pattern to the percentage of metaphase spreads (Figure 2G). The proportions of normal diploid metaphase spreads in 30 samples were 43.3, 60.0 and $46.7 \%$ in L15F, L15FHBNS and L15 complete, respectively (Figure 2H). These data indicate that simple addition of only fetal bovine serum to L15 medium is able to stimulate cellular proliferation followed by metaphase spread preparation. Additionally, when we counted the number of countable metaphase spreads in the L15F group, an average of 30.6 spreads per a sample could be obtained, indicating that a sufficient number of available metaphase spreads for karyotyping can be obtained by this method.

Finally, based on arm length ratios from 20 prepared metaphase spreads, we found that there were ten metacentric $(M)$ and eight submetacentric (SM) chromosomes in 15 


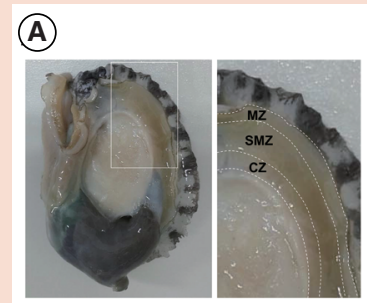

(B)

(C)

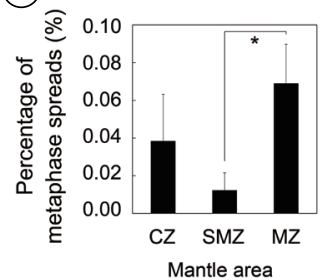

(F)

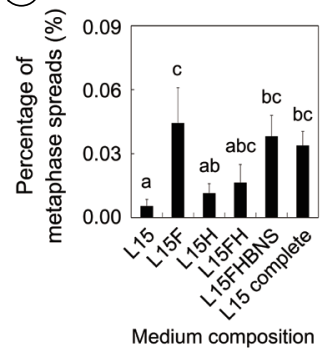

(a)

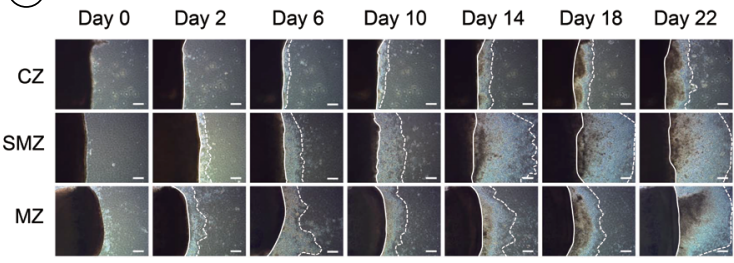

(E)
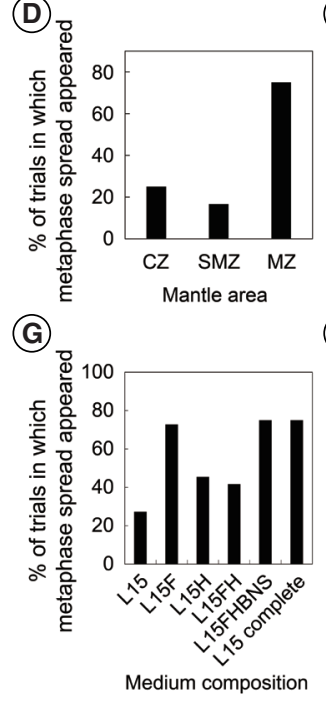

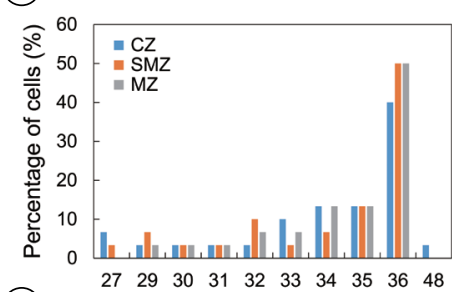

(H)

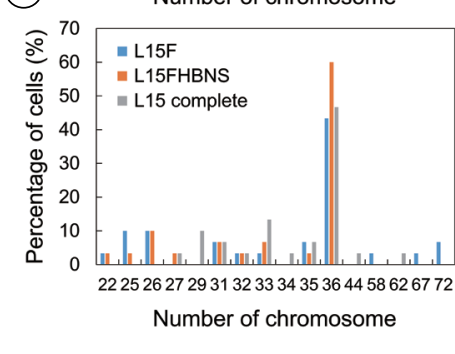

Figure 2. Effects of mantle area and medium composition on metaphase spread preparation by mantle tissue culture. For mantle area test, mantle tissue fragments retrieved from three mantle areas were cultured for 14 or 22 days in complete media in an $18^{\circ} \mathrm{C}$ incubator in air and for medium composition test, MZ-derived tissue fragments were cultured for 14 days in six different media in an $18^{\circ} \mathrm{C}$ incubator in air. After that, the cultured cells were used for metaphase spread preparation. (A) Figures showing three mantle areas including $\mathrm{CZ}, \mathrm{SMZ}$ and $\mathrm{MZ}$. (B) Growth aspects of tissue fragment-derived cell outgrowth depending on mantle area. Proliferation and migration of the cells were observed from day 2 in $\mathrm{CZ}$ and day 6 in SMZ and MZ, and it continued to day 22 in all groups. A relatively high cell growth was observed in SMZ and MZ compared with CZ. Solid and dotted lines indicate the border of mantle tissue fragments and the range of the expanded cells, respectively. Scale bar $=200 \mu \mathrm{m}$. (C) Percentage of metaphase spreads according to mantle area. The percentage in $\mathrm{MZ}$ was significantly higher than that in SMZ ( $p=0.013$, t-test), although the difference was not observed between MZ and CZ. (D) Percentage of trials in which metaphase spread appeared. A significantly high percentage was observed in the MZ group compared with the other groups. (E) Percentage of cells containing normal or abnormal number of chromosomes. Percentage of the cells that have normal chromosome number was 40, 50 and 50\% in the cells derived from CZ, SMZ and MZ, respectively. (F) Percentage of metaphase spreads according to medium composition. Compared to the L15 group, a significantly high frequency was observed in L15F, L15FHBNS and L15 complete groups. ${ }^{a-c}$ Different letters indicate significant differences, at $p<0.05$ [analysis of variance (ANOVA) followed by Duncan test]. (G) Percentage of trials in which metaphase spread appeared. Similar pattern with (F) was shown in this data. (H) Percentage of cells containing normal or abnormal number of chromosomes. Percentage of the cells that have normal chromosome number was 43.3, 60.0 and $46.7 \%$ in the cells derived from L15F, L15FHBNS and L15 complete, respectively. For (C) and (F), data are mean \pm SEM from 12 replicate experiments. BNS: 2-mercaptoethanol, non-essential amino acid, and sodium selenite; CZ: Central zone; F: Fetal bovine serum; H: Hemolymph; L15: Leibovitz's L-15 medium; L15 complete: L15FHBNS plus basic fibroblast growth factor, epidermal growth factor, leukemia inhibitory factor, abalone embryo extract, and medaka embryo extract; MZ: Marginal zone; SEM: Standard error of the mean; SMZ: Sub-marginal zone.

(75\%) metaphase spreads, 9M and 9SM in three (15\%), $11 \mathrm{M}$ and $7 \mathrm{SM}$ in one (5\%), and $12 \mathrm{M}$ and $6 \mathrm{SM}$ in one (5\%). These results are in accordance with previous reports that analyzed the karyotype of $H$. discus hannai [13-15], suggesting that the method established in this study is effective with regard to karyotype analysis as well as the methodological aspect.
Collectively, we established an efficient method (see supplementary protocol) enabling preparation of metaphase spreads without animal sacrifice in adult abalone by using the proliferating cells derived from the culture of mantle tissue fragments. This method can be used for cytogenetic analyses of live adult abalone.

\section{Author contributions}

Y.K.N. and S.P.G. conceived and designed the experiments. J.H.R. and S.Y.L. performed experiments. J.H.R, Y.K.N and S.P.G. analyzed the data. J.H.R. and S.P.G. wrote the paper.

\section{Financial \& competing interests disclosure}

This work was supported by the grant from the Golden Seed Project (GSP), Ministry of Oceans and Fisheries, Republic of Korea. The authors have no other relevant affiliations or financial involvement with any organization or entity with a financial interest in or financial conflict with the subject matter or materials discussed in the manuscript apart from those disclosed. No writing assistance was utilized in the production of this manuscript.

\section{Ethical conduct of research}

The authors state that they have obtained appropriate institutional review board approval or have followed the principles outlined in the Declaration of Helsinki for all human or animal experimental investigations. In addition, for investigations involving human subjects, informed consent has been obtained from the participants involved.

\section{Open access}

This work is licensed under the Creative Commons Attribution 4.0 License. To view a copy of this license, visit http://creativecommons.org/licenses/by/4.0/

\section{Supplementary data}

To view the supplementary data that accompany this paper please visit the website at: www.future-science.com/doi/ suppl/10.2144/btn-2018-0014

\section{References}

1. Cook PA, Gordon HR. World abalone supply, markets, and pricing. J. Shellfish Res. 29, 569571 (2010).

2. Elliott NG. Genetic improvement programmes in abalone: what is the future. Aquac. Res. 31, 51-59 (2000).

3. Minkler J. Chromosomes of the black abalone Haliotis cracherodii. Experientia 33, 1143 (1977).

4. Jarayabhand P, Yom-La R, Popongviwat A. Karyotypes of marine molluscs in the family Haliotidae found in Thailand. J. Shellfish Res. 17, 761-764 (1998). 
5. Pila EA, Sullivan JT, Wu XZ et al. Haematopoiesis in molluscs: a review of haemocyte development and function in gastropods, cephalopods and bivalves. Dev. Comp. Immunol. 58, 119-128 (2016).

6. Rinkevich B. Cell cultures from marine invertebrates: new insights for capturing endless stemness. Mar. Biotechnol. 13, 345-354 (2011).

7. Yoshino TP, Bickham U, Bayne CJ. Molluscan cells in culture: primary cell cultures and cell lines. Can. J. Zool. 91, 391-404 (2013).

8. Colombera D, Tagliaferri F. Chromosomes from male gonads of Haliotis tuberculata and Haliotis lamellosa (Haliotidae, Arche-ogastropoda, Mollusca). Caryologia 36, 231-234 (1983).

9. Auzoux-Bordenave S, Fouchereau-Peron M, Helleouet MN, Doumenc D. CGRP regulates the activity of mantle cells and hemocytes in abalone primary cell cultures (Haliotis tuberculata). J. Shellfish Res. 26, 887-894 (2007).

10. Morash AJ, Alter K. Effects of environmental and farm stress on abalone physiology: perspec- tives for abalone aquaculture in the face of global climate change. Rev. Aquac. 8, 342-368 (2016).

11. Armstrong DA, Armstrong JL, Krassner SM, Pauley GB. Experimental wound repair in the black abalone Haliotis cracherodii. J. Invertebr. Pathol. 17, 216-227 (1971).

12. Ryu JH, Nam YK, Gong SP. Factors affecting primary cultures of abalone Haliotis discus hannai ovary-dissociated cells and general culture aspects. Fish Aquat. Sci. 18, 81-88 (2015).

13. Arai $\mathrm{K}$, Tsubaki H, Ishitani $\mathrm{Y}$, Fujino K. Chromosomes of Haliotis discus hannai INO and $H$. discus REEVE. Bull. Japan Soc. Sci. Fish 48, 1689-1691 (1982).

14. Amar-Basulto G, Lafarga-de la Cruz F, IturraConstant P, Gallardo-Escarate C. Karyotype analysis of interspecific hybrids between Haliotis rufescens and Haliotis discus hannai. Aquac. Res. 42, 1460-1466 (2011).

15. Wang H, Luo X, You W, Dong Y, Ke C. Cytogenetic analysis and chromosomal character- istics of the polymorphic 18S rDNA of Haliotis discus hannai from Fujian, China. PLoS ONE 10, e0113816 (2015)

First draft submitted: 22 February 2018; Accepted for publication: 10 May 2018

Address correspondence to: Seung Pyo Gong; Department of Fisheries Biology, Pukyong National University, Busan 48513, Korea; Tel.: +82 51629 5912; Fax: +82 51629 5908;

E-mail: gongsp@pknu.ac.kr

To purchase reprints of this article contact: s.cavana@future-science.com

\section{Capture Full-Length Genes}

\section{for phased sequencing and structural analysis.}

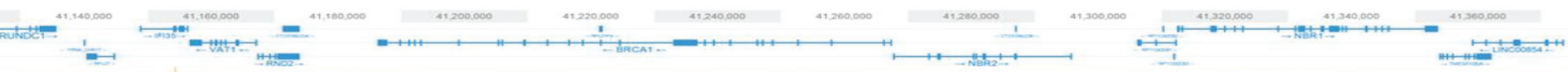

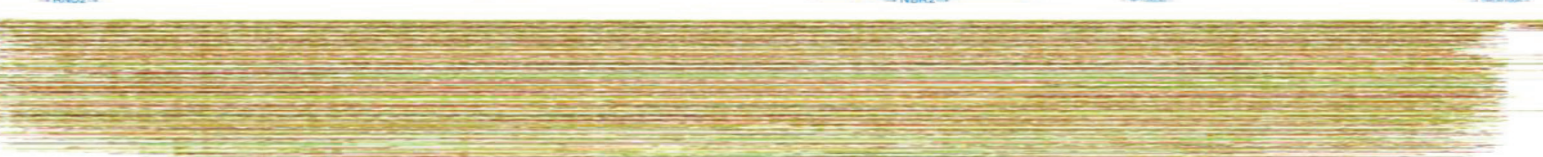

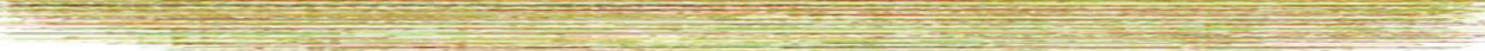

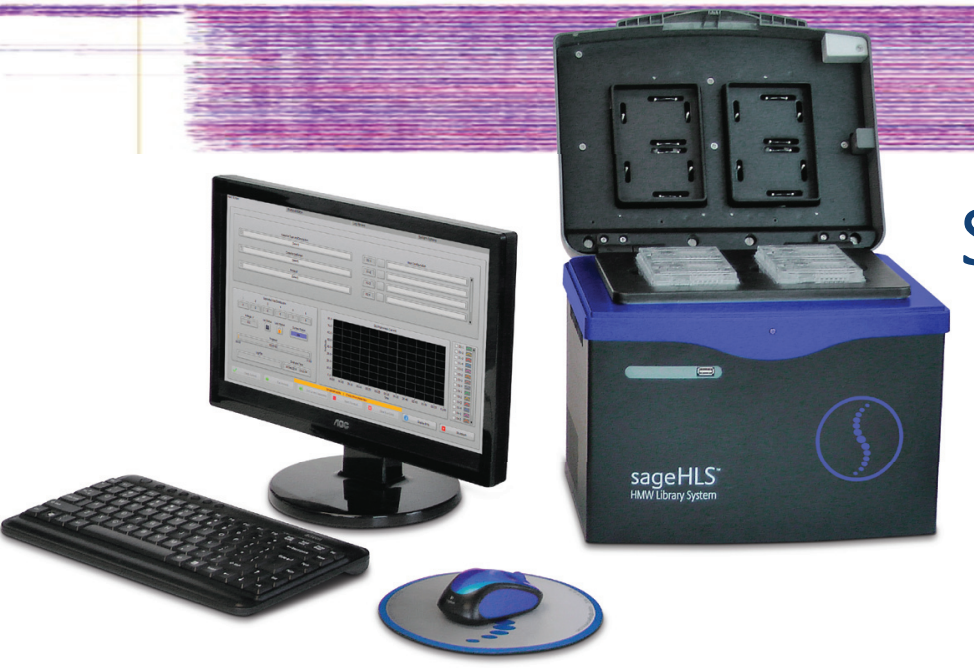

sage HLS HMW Library System

- flank genomic regions with CRISPR/Cas9

- target regions up to $500 \mathrm{~kb}$

- collect multiple targets 